\title{
Divergent predictions of carbon storage between two global land models: attribution of the causes through traceability analysis
}

Rashid Rafique et al.

Correspondence to: Rashid Rafique (rashidbao@gmail.com) and Yiqi Luo (yluo@ ou.edu)

The copyright of individual parts of the supplement might differ from the CC-BY 3.0 licence. 
Supplementary Material

Table 1. Translation of plant functional types from CLM-CASA' to CABLE biomes

\begin{tabular}{cc}
\hline CLM-CASA' PFT & CABLE-compatible biome \\
\hline needleleaf evergreen temperate tree & evergreen needleleaf forest \\
needleleaf evergreen boreal tree & evergreen needleleaf forest \\
needleleaf deciduous boreal tree & deciduous needleleaf forest \\
broadleaf evergreen tropical tree & evergreen broadleaf forest \\
broadleaf evergreen temperate tree & evergreen broadleaf forest \\
broadleaf deciduous tropical tree & deciduous broadleaf forest \\
broadleaf deciduous temperate tree & deciduous broadleaf forest \\
broadleaf deciduous boreal tree & deciduous broadleaf forest \\
broadleaf evergreen shrub & shrubland \\
broadleaf deciduous temperate & shrubland \\
shrub & shrubland \\
broadleaf deciduous boreal shrub & tundra \\
c3 arctic grass & $\mathrm{c3}$ grassland \\
c3 non-arctic grass & c4 grassland \\
c4 grass & \\
\hline \hline
\end{tabular}

